$1-1-2014$

\title{
Decay of helical Kelvin waves on a quantum vortex filament
}

Robert A. Van Gorder

University of Central Florida

Find similar works at: https://stars.library.ucf.edu/facultybib2010 University of Central Florida Libraries http://library.ucf.edu

This Article is brought to you for free and open access by the Faculty Bibliography at STARS. It has been accepted for inclusion in Faculty Bibliography 2010s by an authorized administrator of STARS. For more information, please contactSTARS@ucf.edu.

\section{Recommended Citation}

Van Gorder, Robert A., "Decay of helical Kelvin waves on a quantum vortex filament" (2014). Faculty Bibliography 2010s. 6215.

https://stars.library.ucf.edu/facultybib2010/6215

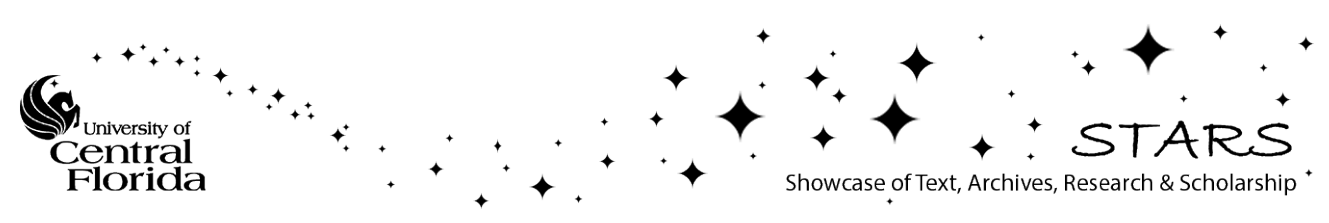




\section{Decay of helical Kelvin waves on a quantum vortex filament}

Robert A. Van Gorder

Citation: Physics of Fluids 26, 075101 (2014); doi: 10.1063/1.4887519

View online: https://doi.org/10.1063/1.4887519

View Table of Contents: http://aip.scitation.org/toc/pht/26/7

Published by the American Institute of Physics

\section{Articles you may be interested in}

Comment on "Motion of a helical vortex filament in superfluid ${ }^{4} \mathrm{He}$ under the extrinsic form of the local induction approximation" [Phys. Fluids 25, 085101 (2013)]

Physics of Fluids 26, 019101 (2014); 10.1063/1.4855296

Motion of a helical vortex filament in superfluid ${ }^{4} \mathrm{He}$ under the extrinsic form of the local induction approximation Physics of Fluids 25, 085101 (2013); 10.1063/1.4816639

Localized-Induction Concept on a Curved Vortex and Motion of an Elliptic Vortex Ring

The Physics of Fluids 8, 553 (1965); 10.1063/1.1761268

Developed quantum turbulence and its decay

Physics of Fluids 24, 011301 (2012); 10.1063/1.3678335

Scattering and leapfrogging of vortex rings in a superfluid

Physics of Fluids 26, 097101 (2014); 10.1063/1.4894745

Solitons on moving space curves

Journal of Mathematical Physics 18, 1654 (1977); 10.1063/1.523453

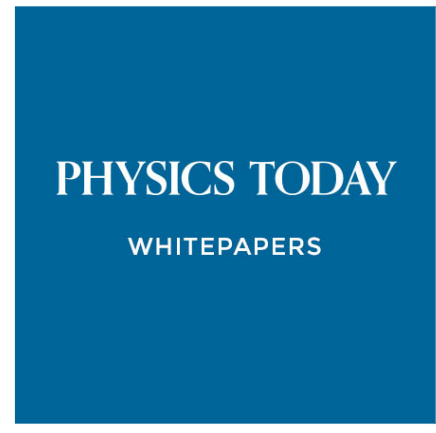

ADVANCED LIGHT CURE ADHESIVES

Take a closer look at what these environmentally friendly adhesive systems can do

\section{READ NOW}

PRESENTED BY Q. MASTERBOND" 


\title{
Decay of helical Kelvin waves on a quantum vortex filament
}

\author{
Robert A. Van Gorder ${ }^{a)}$ \\ Department of Mathematics, University of Central Florida, Orlando, \\ Florida 32816-1364, USA
}

(Received 16 March 2014; accepted 25 June 2014; published online 15 July 2014)

\begin{abstract}
We study the dynamics of helical Kelvin waves moving along a quantum vortex filament driven by a normal fluid flow. We employ the vector form of the quantum local induction approximation (LIA) due to Schwarz. For an isolated filament, this is an adequate approximation to the full Hall-Vinen-Bekarevich-Khalatnikov dynamics. The motion of such Kelvin waves is both translational (along the quantum vortex filament) and rotational (in the plane orthogonal to the reference axis). We first present an exact closed form solution for the motion of these Kelvin waves in the case of a constant amplitude helix. Such solutions exist for a critical wave number and correspond exactly to the Donnelly-Glaberson instability, so perturbations of such solutions either decay to line filaments or blow-up. This leads us to consider helical Kelvin waves which decay to line filaments. Unlike in the case of constant amplitude helical solutions, the dynamics are much more complicated for the decaying helical waves, owing to the fact that the rate of decay of the helical perturbations along the vortex filament is not constant in time. We give an analytical and numerical description of the motion of decaying helical Kelvin waves, from which we are able to ascertain the influence of the physical parameters on the decay, translational motion along the filament, and rotational motion, of these waves (all of which depend nonlinearly on time). One interesting finding is that the helical Kelvin waves do not decay uniformly. Rather, such waves decay slowly for small time scales, and more rapidly for large time scales. The rotational and translational velocity of the Kelvin waves depend strongly on this rate of decay, and we find that the speed of propagation of a helical Kelvin wave along a quantum filament is large for small time while the wave asymptotically slows as it decays. The rotational velocity of such Kelvin waves along the filament will increase over time, asymptotically reaching a finite value. These decaying Kelvin waves correspond to wave number below the critical value for the Donnelly-Glaberson instability, and hence our results on the Schwarz quantum LIA correspond exactly to what one would expect from prior work on the Donnelly-Glaberson instability. (C) 2014 AIP Publishing LLC. [http://dx.doi.org/10.1063/1.4887519]
\end{abstract}

\section{INTRODUCTION}

The motion of a vortex filament in a superfluid was given by the HVBK theory (Hall and Vinen, ${ }^{1,2}$ Bekarevich and Khalatnikov, ${ }^{3}$ Nemirovskii ${ }^{4}$ ). Including the effect of frictional force exerted by the normal fluid on a vortex, the self-induced velocity of a thin vortex filament in the reference frame moving with the superfluid approximated by replacing the Biot-Savart term with the local induction approximation (LIA) is given by $\mathrm{Schwarz}^{5}$

$$
\mathbf{v}=\gamma \kappa \mathbf{t} \times \mathbf{n}+\alpha \mathbf{t} \times(\mathbf{U}-\gamma \kappa \mathbf{t} \times \mathbf{n})-\alpha^{\prime} \mathbf{t} \times(\mathbf{t} \times(\mathbf{U}-\gamma \kappa \mathbf{t} \times \mathbf{n})),
$$

where $\mathbf{U}$ is the normal fluid velocity, $\mathbf{t}$ and $\mathbf{n}$ are the unit tangent and unit normal vectors to the vortex filament, $\kappa$ is the local curvature, $\gamma=\frac{\Gamma}{4 \pi} \ln \left(c / \kappa a_{0}\right)$ is a dimensionless composite parameter $(\Gamma$ is

\footnotetext{
a) Author to whom correspondence should be addressed. Electronic mail: rav@knights.ucf.edu
} 
the dimensionless quantum of circulation, $c$ is a scaling factor of order unity, $a_{0} \approx 1.3 \times 10^{-8} \mathrm{~cm}$ is the effective core radius of the vortex), $\alpha$ and $\alpha^{\prime}$ are dimensionless friction coefficients which are small (except near the $\lambda$-point; for reference, the $\lambda$-point is the temperature $(\approx 2.17 \mathrm{~K})$ below which normal fluid Helium transitions to superfluid Helium ${ }^{6}$ ). The parameter $\gamma$ is assumed to be a constant; that is, we assume that any variation in $\gamma$ with $\kappa$ is small enough to neglect. Schwarz ${ }^{5}$ discusses permissible values of the mutual friction parameters: at temperature $T=1 \mathrm{~K}$ we have $\alpha=0.006$ and $\alpha^{\prime}=0.003$, while at temperature $T=1.5 \mathrm{~K}$ we have $\alpha=0.073$ and $\alpha^{\prime}=0.018$.

The first term on the right-hand side of (1) is exactly the classical LIA $\left(\alpha, \alpha^{\prime}=0\right)$. This is an approximation to the Biot-Savart dynamics, which are given by Boffetta $\mathrm{et} \mathrm{al.}^{7}$

$$
\frac{d \mathbf{r}}{d t}=\frac{\Gamma}{4 \pi} \int \frac{\left(\mathbf{r}_{0}-\mathbf{r}\right) \times d \mathbf{r}_{0}}{\left|\mathbf{r}_{0}-\mathbf{r}\right|^{3}}
$$

where $\Gamma$ is the strength (circulation) of the filament. In the case of a classical helical vortex filament, the LIA can accurately approximate the Biot-Savart dynamics in a qualitative sense. For the quantum case,

$$
\frac{d \mathbf{r}}{d t}=\frac{\Gamma}{4 \pi} \int \frac{\left(\mathbf{r}_{0}-\mathbf{r}\right) \times d \mathbf{r}_{0}}{\left|\mathbf{r}_{0}-\mathbf{r}\right|^{3}}+\alpha \mathbf{t} \times(\mathbf{U}-\gamma \kappa \mathbf{t} \times \mathbf{n})-\alpha^{\prime} \mathbf{t} \times(\mathbf{t} \times(\mathbf{U}-\gamma \kappa \mathbf{t} \times \mathbf{n})) .
$$

The self-interaction is non-local, while the coupling terms of the normal fluid flow with the vortex filament motion due to the mutual friction parameters are local. In the case of a helix, the solutions of (3) can be approximated (in a qualitative sense) by solutions of (1). Note that (1) can be considered in Cartesian or arclength coordinates. Each have their advantage, but we shall consider the Cartesian coordinates sense these allow us to more quickly view the structure of the filaments. In Cartesian coordinates, we write $\mathbf{v}=\frac{d \mathbf{r}}{d t}$ as is common in the literature concerning vortex dynamics in Cartesian coordinates (although note that, in arclength coordinates, one could scale the quantity $v$ differently).

In the present paper, we shall study the motion of helical Kelvin waves along a quantum vortex filament under the Schwarz model (1). Due to the use of the LIA, the model (1) disregards the mutual interaction of vortex filaments and vortex reconnections. The LIA also disregards the interaction of Kelvin waves propagating along the same vortex line, creating energy and other cascades, leading to a decay of Kelvin waves even without mutual frictions. Therefore, the model we use here is useful for studying the dynamics of a regular helical filament on which Kelvin waves propagate in a uniform manner (i.e., without any mutual interaction). We should also state that the LIA best approximates the dynamics of such Kelvin waves when the helix is not tightly coiled (i.e., when the wave number is not too large). Therefore, in the case where the vortex filament is a single helical structure which is not too tightly coiled, we expect that the dynamics obtained from the LIA are an accurate qualitative representation of the dynamics inherent in the non-local Biot-Savart formulation. This is the case we consider in the present paper.

The time evolution of the vortex filament will be described in the Cartesian reference frame, as this permits one to view the filaments directly. There are three main components of the time evolution for such nonlinear waves: translation along a central axis, rotation about the central axis, and decay. In the case of no decay (i.e., the amplitude of the waves remains constant in time, so the waves are eternal), the filament can be described exactly-in closed form. This solution corresponds to the critical wave number for the Donnelly-Glaberson instability. ${ }^{8-11}$ When the Kelvin waves are allowed to decay, which is more physically relevant yet much harder to investigate mathematically, we are able to obtain a dynamical system which governs the time evolution of the translational and rotational motion, in addition to the decay rate. Unlike what may be inferred from the eternal solution, the decay of the Kelvin waves is not constant in time, and we are able to demonstrate mathematically that the rate of decay should gradually increase until the helical Kelvin waves dissipate, leaving a line filament. Numerical simulations verify the analytical results. The influence of the normal fluid velocity, the mutual friction parameters, and the wave number of the Kelvin waves on the time evolution is discussed in detail.

While a number of analytical studies on helical filament structures exist, they can generally be split into two groups. First, there are studies on helical filaments in the classical LIA (see for instance, Refs. 12 and 13), which correspond to the zero temperature limit $\left(\alpha, \alpha^{\prime}=0\right)$. Second, there 
are a number of approximations to the full vector equation (1). Assuming that translational effects are small (or negligible, through a change of the spatial coordinate), one may define a potential form of the quantum LIA. This has been done for the Cartesian ${ }^{14}$ and arclength-tangent ${ }^{16}$ frames. In such formulations, the filaments are assumed to satisfy some constraints (for instance, they should be of sufficient bounded variation-see Ref. 15. As was discussed in Refs. 17 and 18, when one considers such potential forms there are certain limitations, since such a formulation does omit certain features of the solutions when the product $A k$ becomes larger.

The benefit of the present study is that we consider the full vector equation (1) without making simplifying assumptions such as considering a potential formulation. This allows us to take into account strong translation of Kelvin waves along the filament. The amplitudes of such solutions still need to be small enough, but this is physically reasonable, and through the vector formulation we actually learn what small enough really means. Instead of simply stating that $A k \ll 1$, we are able to see exactly what bound on $A k$ one should have in order to obtain helical solutions, including those which decay in time. That is to say, a rather natural bound on the amplitude of solutions can be obtained rather directly from the solutions. The reason that such restrictions exist is a physical one: due to the Donnelly-Glaberson instability mentioned above, the solutions will become unstable if $k$ becomes large. As it turns out, the restriction we obtain from the vector formulation exactly corresponds to the Donnelly-Glaberson instability, lending validity to our approach. Furthermore, we are able to consider decay of the Kelvin waves in time, which is not accessible under potential forms of the LIA. As we shall see, the translation and decay are not constant in time, so the approach taken here is required in order to study such quantum vortex dynamics (one cannot simply assume that the translational velocity or the decay rate are constant in time). From this, we are able to study the decay of helical Kelvin waves along a quantum vortex filament in a logical and coherent manner.

\section{PROPAGATION OF A HELICAL FILAMENT DRIVEN BY THE NORMAL FLUID: THE CONSTANT AMPLITUDE SOLUTION}

Assume that the filament is aligned on the same axis along which the normal fluid is directed. This is not a strong assumption in the case of helical filaments, as it means we choose our coordinates so that one coincides with the central axis of the helix. (For other, more complicated filaments, one may not always be able to have such a nice geometry.) Choosing our geometry in this way, we may write the normal fluid velocity as $\mathbf{U}=(U, 0,0)$ (such a flow configuration can be used to drive Kelvin waves along a line filament) and the vortex filament as $\mathbf{r}=(x, y(x, t), z(x, t))$. A line filament will take the form $\mathbf{r}_{0}=(x, 0,0)$. We shall show that under the Schwarz model, the normal fluid velocity $\mathbf{U}$ induces helical perturbations in the line filament. To do so, we equivalently demonstrate the existence of a helical filament of the form

$$
\mathbf{r}=\left(x+\beta_{0} t, A \cos \left(k\left[x+\beta_{0} t\right]-\phi_{0} t+x_{0}\right), A \sin \left(k\left[x+\beta_{0} t\right]-\phi_{0} t+x_{0}\right)\right)
$$

to the Schwarz model. Here, $A$ is the amplitude, $\beta_{0} t$ is the translation of the central axis along which the filament is aligned due to time, $k$ is the wave number, $\phi_{0}$ is the true frequency, and $x_{0}$ is some constant which may be calibrated subject to any helical initial condition. Let us denote $\eta=k x-\omega_{0} t$ $+x_{0}$, where we set $\omega_{0}=\phi_{0}-k \beta_{0}$ for simplicity. The constant $\omega_{0}$ is the apparent frequency, and it will simplify some calculations to work with $\omega_{0}$ instead of $\phi_{0}$ directly. Of course, we can always recover $\phi_{0}=k \beta_{0}+\omega_{0}$.

We need to convert the right-hand side of (1) into Cartesian coordinates. We compute several quantities needed for the right-hand side of (1):

$$
\begin{gathered}
\frac{d x}{d s}=\frac{1}{\sqrt{1+A^{2} k^{2}}}, \\
\mathbf{t}=\frac{(1,-A k \sin (\eta), A k \cos (\eta))}{\sqrt{1+A^{2} k^{2}}}, \quad \kappa \mathbf{n}=\frac{\left(0,-A k^{2} \cos (\eta),-A k^{2} \sin (\eta)\right)}{\left(1+A^{2} k^{2}\right)}, \\
\kappa \mathbf{t} \times \mathbf{n}=\frac{\left(A^{2} k^{3}, A k^{2} \sin (\eta),-A k^{2} \cos (\eta)\right)}{\left(1+A^{2} k^{2}\right)^{3 / 2}},
\end{gathered}
$$




$$
\begin{gathered}
\mathbf{t} \times(\kappa \mathbf{t} \times \mathbf{n})=-\kappa \mathbf{n}, \quad \mathbf{t} \times[\mathbf{t} \times(\kappa \mathbf{t} \times \mathbf{n})]=-\kappa \mathbf{t} \times \mathbf{n}, \\
\mathbf{t} \times \mathbf{U}=\frac{(0, A k U \cos (\eta), A k U \sin (\eta))}{\sqrt{1+A^{2} k^{2}}}, \\
\mathbf{t} \times(\mathbf{t} \times \mathbf{U})=\frac{\left(-A^{2} k^{2} U,-A k U \sin (\eta), A k U \cos (\eta)\right)}{1+A^{2} k^{2}} .
\end{gathered}
$$

In the above, the quantity $s$ denotes the arc length of the helical filament. We are essentially converting the arc length form of the quantum LIA into a specific Cartesian form in the case of a helical vortex filament. This differs from the study, ${ }^{15}$ where Eq. (1) was first reduced into a potential form (which is an approximation, in that it permits certain types of motion in the case where the helix is of sufficient bounded variation, i.e., $A k$ small enough). In the present vector formulation, no such potential form is assumed, which importantly allows translation of Kelvin waves along the vortex filament. In addition, this formulation permits us to later consider the case where amplitude is non-constant, which we use to study the decay of Kelvin waves along a quantum vortex filament. There will still be restrictions on $A k$, but these will be natural restrictions inherent to the quantum LIA, rather than artificial restrictions due to approximating the LIA. There is actually a physical reason that such a restriction on $A k$ exists. Due to the Donnelly-Glaberson instability, the solutions will become unstable if $k$ becomes large. So, solutions we consider correspond either to this critical value or to values of $k$ below this critical value (see Sec. III). Since the restriction we obtain from the vector formulation used here exactly corresponds to the Donnelly-Glaberson instability, we feel this lends validity to our approach.

As mentioned earlier, the velocity $\mathbf{v}$ of the vortex filament is written as the time derivative of the vortex filament curve if we use Cartesian coordinates, $\mathbf{v}=\mathbf{r}_{t}$. Therefore, the term on the left-hand side of (1) is given by

$$
\mathbf{r}_{t}=\left(\beta_{0}, A \omega_{0} \sin (\eta),-A \omega_{0} \cos (\eta)\right)
$$

Placing these into (1), we obtain three equations, one for each of the $x, y$ and $z$ components. Simplifying these, and noting that the $z$ equation is equivalent to the $y$ equation, we obtain the three parameter restrictions

$$
\begin{aligned}
\beta_{0} & =\frac{\left(1-\alpha^{\prime}\right) \gamma A^{2} k^{3}}{\left(1+A^{2} k^{2}\right)^{3 / 2}}+\frac{\alpha^{\prime} A^{2} k^{2} U}{1+A^{2} k^{2}}, \\
A \omega_{0} & =\frac{\left(1-\alpha^{\prime}\right) \gamma A k^{2}}{\left(1+A^{2} k^{2}\right)^{3 / 2}}+\frac{\alpha^{\prime} A k U}{1+A^{2} k^{2}}, \\
0 & =\frac{\alpha A k U}{\sqrt{1+A^{2} k^{2}}}-\frac{\alpha \gamma A k^{2}}{1+A^{2} k^{2}} .
\end{aligned}
$$

Equation (12) gives the translation of the first coordinate. The remaining two conditions determine the frequency $\omega_{0}$ and the wave number $k$. Compared to Ref. 15, the result for the potential form of the quantum LIA accurately gave the first term for $\omega_{0}$, but the second term involving $U$ is off by a factor, since the term giving this factor was approximated. This was pointed out in Ref. 17. From (14), we find that the wave number must take the critical value

$$
k^{*}=\frac{U}{\sqrt{\gamma^{2}-A^{2} U^{2}}},
$$

which is exactly the critical value for the Donnelly-Glaberson instability. Using (12) and (15) in (13), we find that

$$
\omega_{0}^{*}=\frac{U^{2}}{\gamma^{2}} \sqrt{\gamma^{2}-A^{2} U^{2}} .
$$

Equation (12) should then read $\beta_{0}^{*}=A^{2} k^{*} \omega_{0}^{*}=A^{2} U^{3} \gamma^{-2}$. From this, the frequency is given by

$$
\phi_{0}=k \beta_{0}^{*}+\omega_{0}^{*}=\left(1+A^{2} k^{* 2}\right) \omega_{0}^{*}=\frac{U^{2}}{\sqrt{\gamma^{2}-A^{2} U^{2}}} .
$$


The constant-amplitude helical filament driven by the normal fluid velocity $\mathbf{U}$ is then given by

$$
\mathbf{r}(x, t)=\left(x+\frac{A^{2} U^{3}}{\gamma^{2}} t, A \cos (\eta), A \sin (\eta)\right),
$$

where

$$
\begin{aligned}
\eta & =\frac{U}{\sqrt{\gamma^{2}-A^{2} U^{2}}} x+\frac{U^{2}}{\gamma^{2}} \sqrt{\gamma^{2}-A^{2} U^{2}} t+x_{0} \\
& =\frac{U}{\sqrt{\gamma^{2}-A^{2} U^{2}}}\left\{x+\frac{A^{2} U^{3}}{\gamma^{2}} t\right\}+\frac{U^{2}}{\sqrt{\gamma^{2}-A^{2} U^{2}}} t+x_{0} .
\end{aligned}
$$

A necessary condition for the existence of such a helical vortex filament solution to the quantum LIA is that $A<\gamma /|U|$, so the amplitude of the helical perturbations to the line filament must be bounded.

In either the small amplitude or small $U$ limit, the vortex filament (19) collapses to a line filament. So, it is clear that the helical perturbations that rise along the line filament are driven by the normal fluid impinging on the superfluid. The normal fluid flow also induces a drift, which manifests in the $t$-dependence of the $x$-coordinate of (19).

Note that such filaments solutions are eternal, in the sense that they do not decay. These solutions correspond to the onset of the Donnelly-Glaberson instability of Kelvin waves driven by the normal fluid flow. The critical value of $k=k^{*}$ therefore corresponds to the critical wave number for this instability. Such helical structures, while eternal, should be unstable. This means that, under small perturbations, the helical filaments will not maintain their form. This makes sense, in light of the fact that there are dissipation effects (such as the friction parameters) in contrast to the driving force due to the normal fluid flow. At $k=k^{*}$, these effects are balanced, whereas under a small perturbation one or the other effects on the vortex filament may dominate.

Temporal growth of a line filament resulting from the Donnelly-Glaberson instability is precisely the mechanism responsible for the formation of the vortex tangle. ${ }^{19}$ The decay of a filament into a line filament (that is, the dissipation of the helical waves along the filament) is physically relevant and should be accounted for. Therefore, we should generalize the particular solution in this section (corresponding to the critical wave number for the Donnelly-Glaberson instability) to account for temporal effects. In order to obtain solutions which exhibit both translational and rotational motion in addition to decay, a more complicated analysis is required, and we perform this analysis in Sec. III.

Giving credit where credit is due, note that the formula for $k^{*}$ given in (15) is that of Ref. 17, although the value of $k^{*}$ was derived in a different manner. (The result of Ref. 15 was an approximation to (15), valid for $A k \ll 1$. So, when $A k$ becomes large, the agreement between (15) and results in Ref. 15 break down. In the valid region, where the helix is sufficiently bounded in variation, the formulas give the same qualitative information.) Importantly, the authors of Ref. 17 obtain (15) by use of a potential formulation (derived without some simplifying assumptions of Ref. 15). Such a potential formulation is not valid for time-variable amplitude helical filaments (as pointed out in Ref. 18), so a different approach (like the one outlined in this paper) is needed. Indeed, in order to account for decay or growth of the helix radius, one needs to maintain the full vector form of the quantum LIA. This is demonstrated in Sec. III, where we are able to consider a formulation which permits growth or decay of the Kelvin waves.

\section{DECAY OF KELVIN WAVES ALONG A QUANTUM VORTEX FILAMENT}

The most interesting part of this paper is to demonstrate the decay of helical Kelvin waves in time. The solution in Sec. II is interesting, because it replicates the constant-amplitude solutions which occur as critical solutions at the onset of the Donnelly-Glaberson instability. However, the more physically interesting case is when one permits the helical Kelvin waves to decay. This is much more mathematically challenging, yet still some results are possible. We shall give both large and 
small time approximations, along with numerics for all times (which agree with the analytics, where the analytics are valid).

The solution obtained in Sec. II does not decay in time: the motion of the helical vortex filament is rotational and translational, but there is no dissipation of the helical waves. In order to account for the decay, it may be tempting to simply include terms of the form $\exp (-r t)$, where $r$ is some positive constant, to (18) (multiplying the sine and cosine terms). (This error was made in a derivation in Ref. 17, where a result was attempted for the decaying helical filament.) Unfortunately, things are not so simple: doing so, one obtains factors of $\exp (-2 r t)$ in the expressions for $\omega$, implying that $\omega$ is not a constant parameter. In order to avoid such contradictions, we must assume that $\omega$ is not a constant in time, nor do we assume the decay rate is constant in time. To this effect, let us consider a filament solution of the form

$$
\mathbf{r}(x, t)=(x+\beta(t), A \exp (-\mu(t)) \cos (v(x, t)), A \exp (-\mu(t)) \sin (v(x, t))),
$$

where $v(x, t)=k[x+\beta(t)]-\phi(t)+x_{0}$. Here $\frac{d \phi}{d t}$ is the true rotational velocity whereas $\frac{d \beta}{d t}$ gives the change in the spatial position with respect to time. Let us define $\omega(t)=\phi(t)-k \beta(t)$. Then $\frac{d \omega}{d t}$ is the apparent rotational velocity, and $v(x, t)=k x-\omega(t)+x_{0}$. It will be simpler to work with $\omega(t)$ than $\phi(t)$ directly, since it will allow us to write independent equations for $\beta(t)$ and $\omega(t)$. After these functions are solved, one may obtain the true rotational velocity through writing $\phi(t)=\omega(t)+k \beta(t)$ and then finding $\frac{d \phi}{d t}=\frac{d \omega}{d t}+k \frac{d \beta}{d t}$.

Following similar derivations to those in Sec. II, we find that such a vortex filament solution (20) exists provided that

$$
\begin{gathered}
\frac{d \beta}{d t}=\left\{\frac{\left(1-\alpha^{\prime}\right) \gamma A^{2} k^{3}}{\left(1+A^{2} k^{2} \exp (-2 \mu(t))\right)^{3 / 2}}+\frac{\alpha^{\prime} A^{2} k^{2} U}{1+A^{2} k^{2} \exp (-2 \mu(t))}\right\} \exp (-2 \mu(t)), \\
\frac{d \omega}{d t}=\frac{\left(1-\alpha^{\prime}\right) \gamma k^{2}}{\left(1+A^{2} k^{2} \exp (-2 \mu(t))\right)^{3 / 2}}+\frac{\alpha^{\prime} k U}{1+A^{2} k^{2} \exp (-2 \mu(t))}, \\
\frac{d \mu}{d t}=\frac{\alpha \gamma k^{2}}{1+A^{2} k^{2} \exp (-2 \mu(t))}-\frac{\alpha k U}{\sqrt{1+A^{2} k^{2} \exp (-2 \mu(t))}} .
\end{gathered}
$$

Equations (21)-(23) constitute a system of differential equations governing the translational $\left(\frac{d \beta}{d t}\right)$, rotational $\left(\frac{d \omega}{d t}\right)$, and decay $\left(\frac{d \mu}{d t}\right)$ effects on the helix in the quantum LIA. The first two of these equations give us

$$
\begin{gathered}
\beta(t)=A^{2} k \int_{0}^{t} \frac{d \omega}{d t}(\tau) \exp (-2 \mu(\tau)) d \tau \\
\omega(t)=\int_{0}^{t}\left\{\frac{\left(1-\alpha^{\prime}\right) \gamma k^{2}}{\left(1+A^{2} k^{2} \exp (-2 \mu(t))\right)^{3 / 2}}+\frac{\alpha^{\prime} k U}{1+A^{2} k^{2} \exp (-2 \mu(t))}\right\} d \tau .
\end{gathered}
$$

Hence, provided we can find $\mu(t)$, the quantities $\beta(t)$ and $\omega(t)$ are able to be calculated.

From (23), we can separate variables and obtain an implicit relation for $\mu(t)$ :

$$
\int_{0}^{\mu(t)} \frac{1+A^{2} k^{2} \exp (-2 w)}{\gamma k^{2}-k U \sqrt{\left.1+A^{2} k^{2} \exp (-2 w)\right)}} d w=\alpha t .
$$

Note that when $\alpha=0$, the decay rate is zero. Therefore, the helical solutions to the classical LIA do not decay in the manner described here.

In the case where the amplitude is very small, $A \ll 1$, we ignore the order $A^{2}$ terms, obtaining $\beta(t)=\bar{\beta}$ (a constant, meaning that there is no translation in time). We then find that

$$
\omega(t)=\left\{\left(1-\alpha^{\prime}\right) \gamma k^{2}+\alpha^{\prime} k U\right\} t,
$$

and

$$
\mu(t)=\alpha k(\gamma k-U) t
$$


From the latter, we see that a necessary condition for decay of the filament is $k>U / \gamma$ whereas the filament will amplify if $k<U / \gamma$. The true rotational motion is given by

$$
\phi(t)=k \bar{\beta}+\left\{\left(1-\alpha^{\prime}\right) \gamma k^{2}+\alpha^{\prime} k U\right\} t,
$$

and thus the true rotational velocity is $\frac{d \phi}{d t}=\frac{d \omega}{d t}=\left(1-\alpha^{\prime}\right) \gamma k^{2}+\alpha^{\prime} k U$.

\section{A. Properties of the decay term $\mu(t)$}

Assume that $\gamma>|U|$. Then, (23) has an equilibrium $\mu^{*}$ which is given by the formula

$$
\mu^{*}=\frac{1}{2} \ln \left(\frac{U^{2}}{\gamma^{2}-\left(1+A^{2}\right) U^{2}}\right) .
$$

In order to $\mu^{*}$ to exist and be positive, we must have the condition

$$
\frac{\gamma^{2}-2 U^{2}}{U^{2}}<A^{2}<\frac{\gamma^{2}-U^{2}}{U^{2}}
$$

on the initial amplitude $A$. As may be verified easily, the positive equilibrium $\mu^{*}$ is always unstable.

Even without the condition $\gamma>|U|$, the function $\mu(t)$ can be shown to increase under reasonable conditions. From the form of (23), if the right-hand side of (23) is initially positive, then it should remain positive for all time. Since we take $\mu(0)=0$, this means that we should have

$$
\frac{\alpha \gamma k^{2}}{1+A^{2} k^{2}}-\frac{\alpha k U}{\sqrt{1+A^{2} k^{2}}}>0, \quad \text { or, equivalently, } \quad \frac{k}{\sqrt{1+A^{2} k^{2}}}>\frac{U}{\gamma} .
$$

For small time, we have that $\mu(t)$ is approximately given by

$$
\mu(t) \approx \alpha k\left(\frac{\gamma k-U \sqrt{1+A^{2} k^{2}}}{1+A^{2} k^{2}}\right) t .
$$

Since $\mu(t)$ is increasing in time, we should have that $\exp (-2 \mu(t)) \rightarrow 0$ as $t \rightarrow \infty$. This is equivalent to taking the amplitude to zero, so in this limit the solutions is equivalent to (28). In Figure 1, we plot a numerical solution to (23) in addition to the small-time approximation (33) and the large-time approximation (28). We see that the approximation (33) is reasonable for $t<\alpha^{-1}$, whereas the approximation (28) is a good fit to the true solution provided $t>\alpha^{-1}$. These solutions indicates an interesting facet of the decay of the helical filament, namely, that the exponential rate of decay is not constant. Instead, the filament decays more slowly for small time values and decays more rapidly for large time. This suggests that such vortex filaments would be observed on small time scales, before decaying to line filaments when time increases.

\section{B. The case of vanishing normal fluid velocity}

In the small $U$ limit, note that we obtain

$$
\frac{d \mu}{d t}=\frac{\alpha \gamma k^{2}}{1+A^{2} k^{2} \exp (-2 \mu(t))},
$$

which admits the exact solution

$$
\mu(t)=\frac{1}{2} \text { LambertW }\left(A^{2} k^{2} \exp \left(A^{2} k^{2}-2 \alpha \gamma k^{2} t\right)\right)+\alpha \gamma k^{2} t-\frac{1}{2} A^{2} k^{2} .
$$

The LambertW function is a special function defined implicitly by the relation

$$
\text { LambertW }(\xi) \exp (\text { LambertW }(\xi))=\xi .
$$

The solution (35) is approximately linear (as seen by plotting the first term), and an accurate linear approximation is given by

$$
\mu(t) \approx \frac{\alpha \gamma k^{2}}{1+A^{2} k^{2}} t
$$




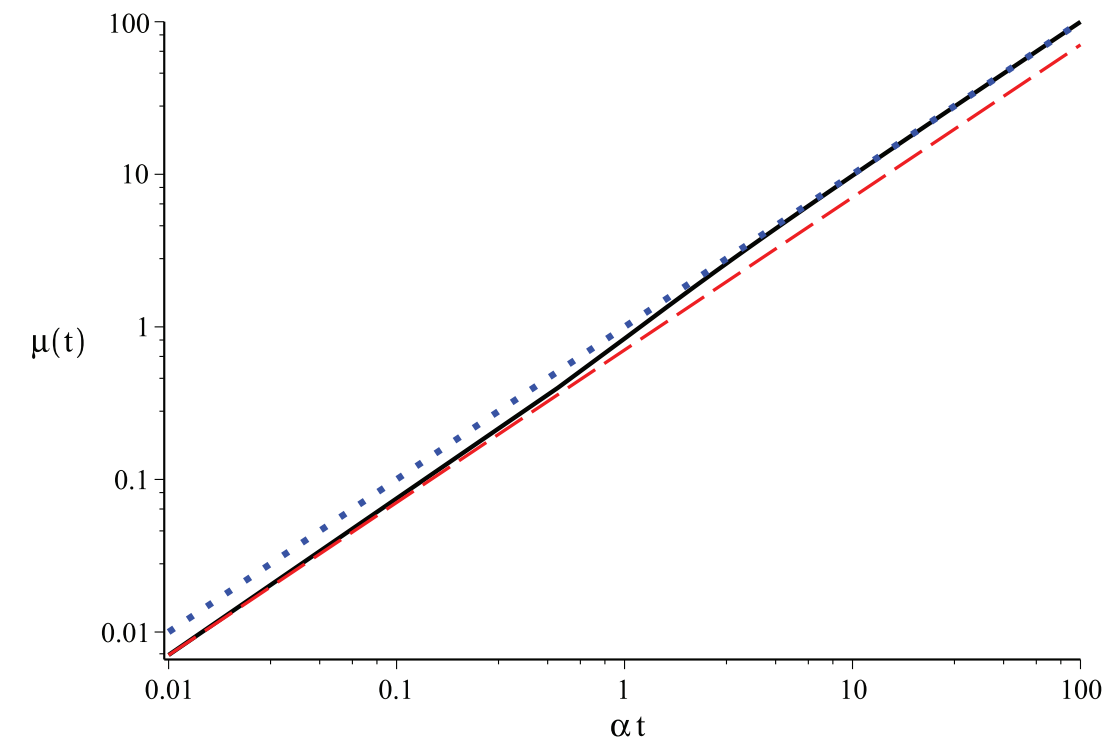

Numerical solution -- Small $\mathrm{t}$ asymptotics " " " " Large $\mathrm{t}$ asymptotics

FIG. 1. Plot of the numerical solution for $\mu(t)$ governed by Eq. (23) along with the small time asymptotic solution (33) and the large time asymptotic solution (28). Parameter values are fixed at $A=0.5, k=U=1, \gamma=2$. For small time $\left(t<\alpha^{-1}\right)$, the approximation (33)) is a good fit to the numerical solution, whereas when time is large $\left(t>\alpha^{-1}\right)$, the approximation (28) accurately describes the numerical solution. With this, we find that helical perturbations along the line vortex filament decay slowly on small time scales, and then more rapidly for larger time scales.

When $U=0$, (22) reduces to

$$
\frac{d \omega}{d t}=\frac{\left(1-\alpha^{\prime}\right) \gamma k^{2}}{\left(1+A^{2} k^{2} \exp (-2 \mu(t))\right)^{3 / 2}}=\frac{1-\alpha^{\prime}}{\alpha} \frac{1}{\sqrt{1+A^{2} k^{2} \exp (-2 \mu(t))}} \frac{d \mu}{d t} .
$$

Integrating both sides of this equation once, and performing relevant algebraic manipulations, we find

$$
\omega(t)=\frac{1-\alpha^{\prime}}{\alpha} \mu(t)+\frac{1-\alpha^{\prime}}{\alpha} \ln \left\{\frac{1+\sqrt{1+A^{2} k^{2} \exp (-2 \mu(t))}}{1+\sqrt{1+A^{2} k^{2}}}\right\} .
$$

Obtaining the linear approximation like that of (37), we have

$$
\omega(t) \approx \frac{\left(1-\alpha^{\prime}\right) \gamma k^{2}}{\left(1+A^{2} k^{2}\right)^{3 / 2}} t,
$$

which is completely consistent with the dispersion relation obtained under LIA for a standard fluid when we set $\alpha^{\prime}=0$.

When $U=0$, the translation effects (21) should be given by

$$
\frac{d \beta}{d t}=\frac{\left(1-\alpha^{\prime}\right) \gamma A^{2} k^{3}}{\left(1+A^{2} k^{2} \exp (-2 \mu(t))\right)^{3 / 2}} \exp (-2 \mu(t))=\frac{1-\alpha^{\prime}}{\alpha} \frac{A^{2} k \exp (-2 \mu(t))}{\sqrt{1+A^{2} k^{2} \exp (-2 \mu(t))}} \frac{d \mu}{d t},
$$

which gives a solution of the form

$$
\beta(t)=\frac{1-\alpha^{\prime}}{\alpha k}\left\{\sqrt{1+A^{2} k^{2}}-\sqrt{1+A^{2} k^{2} \exp (-2 \mu(t))}\right\} .
$$

Up to first order, this solution is approximated like

$$
\beta(t) \approx \frac{\left(1-\alpha^{\prime}\right) \gamma A^{2} k^{3}}{\left(1+A^{2} k^{2}\right)^{3 / 2}} t
$$


when $t$ is small enough. Setting $\alpha^{\prime}=0$, we again recover the expected result from the standard fluid LIA. When $t$ is large, we should have $\exp (-2 \mu(t)) \rightarrow 0$ and therefore

$$
\beta(t) \approx \frac{1-\alpha^{\prime}}{\alpha k}\left\{\sqrt{1+A^{2} k^{2}}-1\right\} .
$$

Regarding the true rotational velocity, under the linear approximations in (40) and (43), we find

$$
\phi(t) \approx k \frac{\left(1-\alpha^{\prime}\right) \gamma A^{2} k^{3}}{\left(1+A^{2} k^{2}\right)^{3 / 2}} t+\frac{\left(1-\alpha^{\prime}\right) \gamma k^{2}}{\left(1+A^{2} k^{2}\right)^{3 / 2}} t=\frac{\left(1-\alpha^{\prime}\right) \gamma k^{2}}{\sqrt{1+A^{2} k^{2}}} t .
$$

Therefore, the true rotational velocity should be approximated like

$$
\frac{d \phi}{d t} \approx \frac{\left(1-\alpha^{\prime}\right) \gamma k^{2}}{\sqrt{1+A^{2} k^{2}}}
$$

for small $t$.

What we find is that, when the effects of the normal fluid velocity are negligible $(U=0)$, for small timescales we obtain the helical filament

$$
\begin{aligned}
\mathbf{r}(x, t) \approx & \left(x+\frac{\left(1-\alpha^{\prime}\right) \gamma A^{2} k^{3}}{\left(1+A^{2} k^{2}\right)^{3 / 2}} t\right) \mathbf{i}_{x}+A \exp \left(-\frac{\alpha \gamma k^{2}}{1+A^{2} k^{2}} t\right) \cos \left(k x-\frac{\left(1-\alpha^{\prime}\right) \gamma k^{2}}{\left(1+A^{2} k^{2}\right)^{3 / 2}} t\right) \mathbf{i}_{y} \\
& +A \exp \left(-\frac{\alpha \gamma k^{2}}{1+A^{2} k^{2}} t\right) \sin \left(k x-\frac{\left(1-\alpha^{\prime}\right) \gamma k^{2}}{\left(1+A^{2} k^{2}\right)^{3 / 2}} t\right) \mathbf{i}_{z},
\end{aligned}
$$

which gradually decays to a line filament with exponential rate of decay $\frac{\alpha \gamma k^{2}}{1+A^{2} k^{2}}=O(\alpha)$ as time increases. Quantum vortex filaments in the $U=0$ case are still physically relevant, ${ }^{20-22}$ and the results here show that small helical excitations along such filaments should still decay despite the absence of the normal fluid.

The normal fluid can result in decay when there are friction parameters (otherwise the quantum vortex filament is not coupled to the normal fluid flow). Thus, zero mutual friction parameters imply there will be no influence of the normal fluid flow on the quantum filament, which is essentially the classical case. On the other hand, just because the normal fluid velocity is zero does not mean that the mutual friction parameters will not result in decay. Indeed, the case of $U=0$ is that of a quantum filament with only self-induced motion. However, in the presence of mutual friction, the waves along the filament should gradually spin down and dissipate (the helical filament will mathematically decay into a quantum filament) even in the absence of a normal fluid flow.

\section{The role of normal fluid velocity on vortex motion and persistence}

While exact solutions for $\mu(t)$ are not forthcoming in the presence of a non-zero normal fluid velocity, we observe that the solutions should be approximately linear, with the slope gradually increasing as $t$ increases (as discussed above). For small $t\left(t<\alpha^{-1}\right)$, we obtain

$$
\begin{aligned}
& \beta(t) \approx\left\{\frac{\left(1-\alpha^{\prime}\right) \gamma A^{2} k^{3}}{\left(1+A^{2} k^{2}\right)^{3 / 2}}-\frac{\alpha^{\prime} A^{2} k^{2} U}{1+A^{2} k^{2}}\right\} t, \\
& \omega(t) \approx\left\{\frac{\left(1-\alpha^{\prime}\right) \gamma k^{2}}{\left(1+A^{2} k^{2}\right)^{3 / 2}}-\frac{\alpha^{\prime} k U}{1+A^{2} k^{2}}\right\} t, \\
& \mu(t) \approx\left\{\frac{\alpha \gamma k}{1+A^{2} k^{2}}-\frac{\alpha k U}{\sqrt{1+A^{2} k^{2}}}\right\} t .
\end{aligned}
$$

We then find that

$$
\phi(t)=\left\{\frac{\left(1-\alpha^{\prime}\right) \gamma k^{2}}{\sqrt{1+A^{2} k^{2}}}-\alpha^{\prime} k U\right\} t
$$




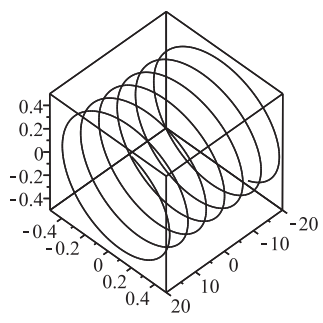

(a)

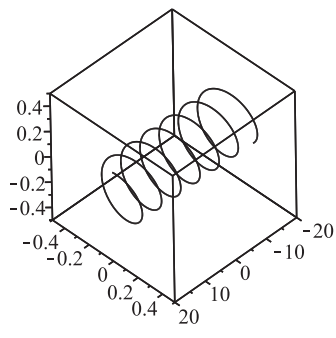

(b)

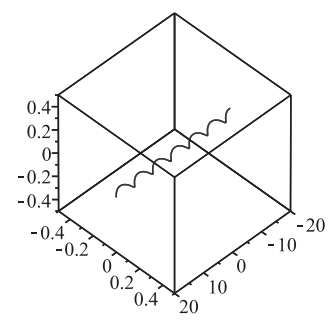

(c)

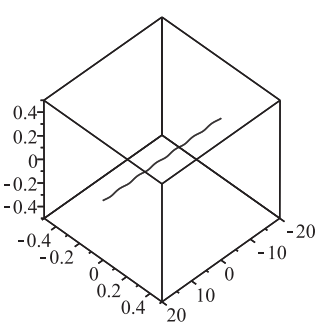

(d)

FIG. 2. Plot of the time evolution of a helical filament solution corresponding to $A=0.5, \gamma=2, k=1$, T=1K (i.e., $\alpha=0.006, \alpha^{\prime}=0.003$ ) and $U=0$. Times referenced are (a) $t=0$, (b) $t=100$, (c) $t=300$, and (d) $t=500$.

So, in small timescales the translational velocity goes as

$$
\frac{d \beta}{d t} \approx \frac{\left(1-\alpha^{\prime}\right) \gamma A^{2} k^{3}}{\left(1+A^{2} k^{2}\right)^{3 / 2}}-\frac{\alpha^{\prime} A^{2} k^{2} U}{1+A^{2} k^{2}},
$$

while the true rotational velocity goes like

$$
\frac{d \phi}{d t} \approx \frac{\left(1-\alpha^{\prime}\right) \gamma k^{2}}{\sqrt{1+A^{2} k^{2}}}-\alpha^{\prime} k U
$$

For large $t\left(t>\alpha^{-1}\right.$ ), we have the approximations $\beta(t) \approx \bar{\beta}$ (a constant, hence there is no transnational motion in the large timescales, so $\bar{\beta})$ denotes the limit $\beta(t) \rightarrow \bar{\beta}$ as $t \rightarrow \infty), \omega(t) \approx\left\{\left(1-\alpha^{\prime}\right) \gamma k^{2}+\right.$ $\left.\alpha^{\prime} k U\right\} t$, and $\mu(t) \approx \alpha k(\gamma k-U) t$ (which is exactly what we found previously, in the small amplitude limit).

Both attributes of the vortex motion, namely, translation and rotation, are increased when we increase the normal fluid velocity. Increasing the normal fluid velocity, we see that solutions will decay more slowly, and therefore persist for longer periods of time. Both of these observations make sense in light of the fact that the normal fluid velocity is expected to drive the vortex. Therefore, increasing the normal fluid velocity results in faster-moving and longer-lasting helical vortex filaments.

In Figure 2, we plot the time evolution of a helical vortex filament as described by the solution of the system of nonlinear differential equations (21)-(23). We take the normal fluid velocity to be zero. In Figure 3 we do the same, while taking a non-zero normal fluid velocity.

From Figure 4(a), we see that the translational motion of the helical Kelvin waves along the vortex filament is strongly influenced by both the normal fluid velocity and the magnitude of the mutual friction parameters. Naturally, in increase in the normal fluid velocity results in an increase in the rate of propagation of a helical Kelvin wave along the $x$-axis. On the other hand, an increase

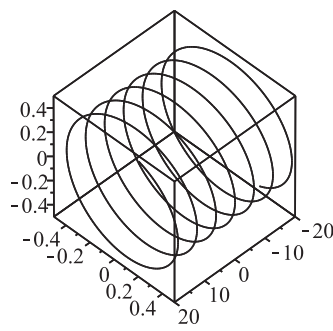

(a)

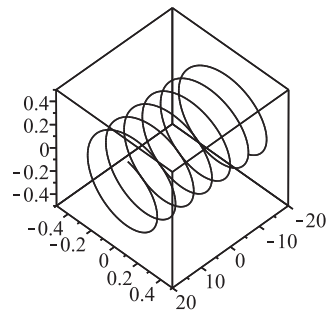

(b)

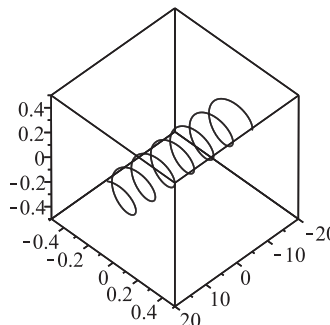

(c)

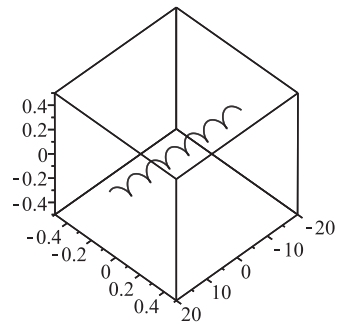

(d)

FIG. 3. Plot of the time evolution of a helical filament solution corresponding to $A=0.5, \gamma=2, k=1$, T=1K (i.e., $\alpha$ $=0.006, \alpha^{\prime}=0.003$ ) and $U=1$. Times referenced are (a) $t=0$, (b) $t=100$, (c) $t=300$, and (d) $t=500$. Note that the decay of the helical perturbations along the filament is markedly slower than in the case of $U=0$ (which was considered in Figure 2). This is because the helical Kelvin waves are driven by the normal flow. 


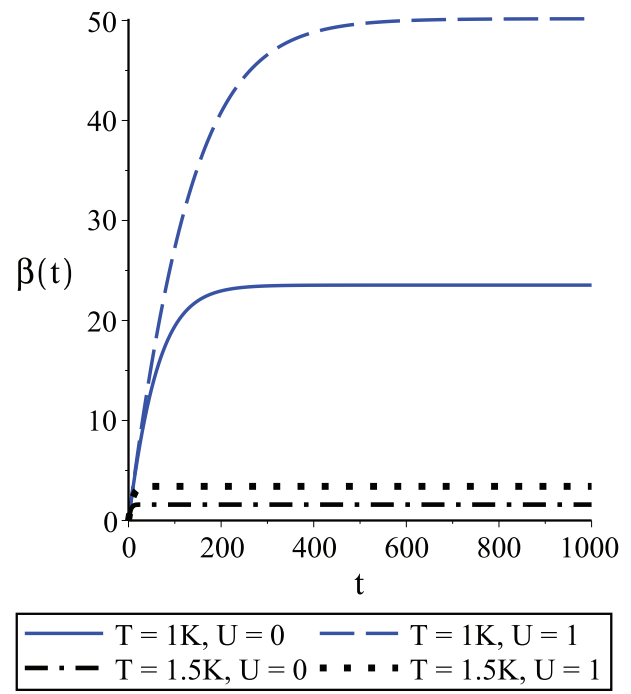

(a)

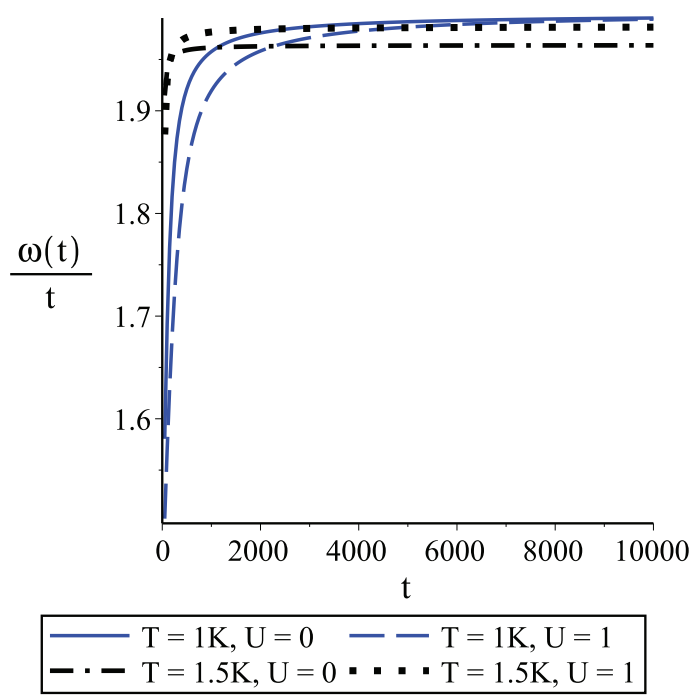

(b)

FIG. 4. Plot of the (a) translation $\beta(t)$ of the $x$-coordinate (the translational motion of the helical waves along the filament) and (b) the effective frequency $\omega(t) / t$ for a helical filament solution corresponding to $A=0.5, \gamma=2, k=1$, for various temperatures and normal fluid velocity. The temperature $\mathrm{T}=1 \mathrm{~K}$ correspond to $\alpha=0.006, \alpha^{\prime}=0.003$, while $\mathrm{T}=1.5 \mathrm{~K}$ corresponds to $\alpha=0.073, \alpha^{\prime}=0.018$.

in the mutual friction parameters slows the propagation of such helical Kelvin waves. While these results are completely intuitive, an analysis of the nonlinear system (21)-(23) is needed to justify these conclusions for a filament in the LIA. Meanwhile, the effective frequency $(\omega(t) / t)$ is not strongly influenced by the normal fluid velocity, nor is it strongly influenced by the mutual friction parameters. This follows from the fact that the leading order term in the dispersion relation (22) does not depends on either $\alpha, \alpha^{\prime}$, or on $U$, and the only term involving $U$ has the multiplication $\alpha^{\prime} U$ so that any effects of $U$ are small.

\section{A DYNAMICAL SYSTEMS APPROACH TO STUDY THE DECAY RATE OF KELVIN WAVES}

In Sec. III, we reduced the equation of motion for a quantum vortex filament into a dynamical system (21)-(23) governing three time-dependent parameters of Kelvin waves which are allowed to decay. A number of properties of the solutions were determined analytically for both large and small time, and numerical results were demonstrated for arbitrary time. From this, we can infer much about the behavior of these decaying Kelvin waves. In this section, we shall take a dynamical systems approach to study the qualitative behavior of this system.

Let us define the function

$$
\theta(t)=\left(1+A^{2} k^{2} \exp (-2 \mu(t))\right)^{-1 / 2} .
$$

Then, Eq. (23) is put into the form

$$
\frac{d \theta}{d t}=\alpha \gamma k^{2} F(\theta)
$$

where

$$
F(\theta)=\theta^{2}(\theta-m)\left(1-\theta^{2}\right)
$$

and with $m$ we denote the constant

$$
m=\frac{U}{k \gamma} .
$$




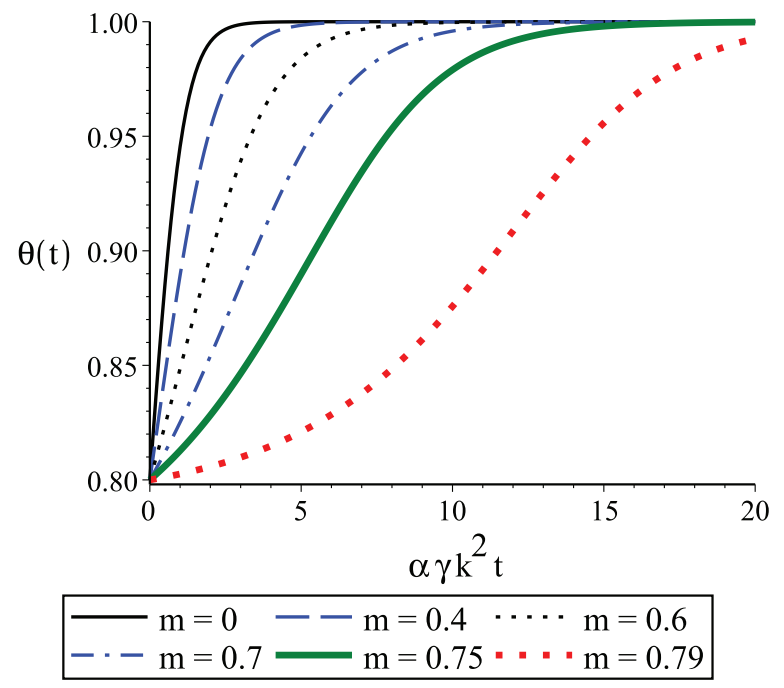

FIG. 5. Plots of the numerical solution profiles for (55) subject to (58). We plot the solutions over the scaled time $\alpha \gamma k^{2} t$, and in all plots we take $\theta(0)=0.8$ so that the plots are all comparable. The smaller the value of $m=\frac{U}{k \gamma}$, the faster the solutions approach the equilibrium value of 1 .

Note that

$$
\theta(0)=\frac{1}{\sqrt{1+A^{2} k^{2}}},
$$

and thus (55) and (58) define an initial value problem for the unknown function $\theta(t)$. The reaction function $F(\theta)$ is a polynomial in $\theta$, hence the analysis of (55) will be a bit simpler than that of (21)-(23).

Observe that

$$
\theta(0)-m=\frac{1}{\sqrt{1+A^{2} k^{2}}}-\frac{U}{k \gamma}>0,
$$

where the inequality follows from (32). Furthermore, clearly $\theta(0)<1$ for all $A, k>0$. Therefore, the initial condition satisfies $m<\theta(0)<1$. Yet, $F(\theta(0))>0$, so the derivative of $\theta$ at $t=0$ is necessarily positive. Indeed, for any $\xi$ satisfying $\theta(0)<\xi<1$, we have $F(\xi)>0$. What this means is that $\theta(t)$ increases until it attains the value $\theta(t)=1$.

$F(\theta)$ has roots $\theta=0, m, 1$, so these are the possible equilibrium values for the dynamics of (55). We find that $F^{\prime}(0)=0, F^{\prime}(m)>0$, and $F^{\prime}(1)<0$. So, by the linear stability test, we conclude that the equilibrium $\theta=m$ is linearly unstable whereas the equilibrium $\theta=1$ is linearly stable. So, $\theta(t) \rightarrow$ 1 as $t \rightarrow \infty$. Yet, since $F(\theta(t))>0$ for all $\theta(0)<\theta(t)<1$, we have that $\theta(t)$ must be increasing for all $t>0$. Therefore, the unknown function $\theta(t)$ increases monotonically from a minimum of $\theta(0)$ at $t=0$ toward an asymptotic value of 1 as $t \rightarrow \infty$. To graphically demonstrate the behavior of $\theta(t)$, we plot numerical solution profiles for (55) subject to (58) in Figure 5. We see that for small $m$, the solution profiles rapidly tend to the equilibrium value of 1 , whereas when $m$ approaches the value $\theta(0)$ (from below, since $m<\theta(0)$ ) the solution profiles more slowly approach the equilibrium value.

With the qualitative behavior of $\theta(t)$ known, let us return to $\mu(t)$. Inverting the relation (54), we should have

$$
\mu(t)=\ln (A k)+\ln \sqrt{\frac{\theta(t)^{2}}{1-\theta(t)^{2}}} .
$$

For finite $t$, this representation is well-defined, as $\theta(0)<\theta(t)<1$ for all finite $t$. However, as $t \rightarrow$ $\infty$, we have that the second logarithm term blows up toward infinity. However, this is completely consistent with what we saw in Sec. III, as $\mu(t)$ was seen to increase without bound for large $t$. It is 
perhaps more useful to rewrite (23) in terms of $\theta(t)$. Doing so, we find that

$$
\frac{d \mu}{d t}=\alpha \gamma k^{2} \theta(t)(\theta(t)-m) \text {. }
$$

It is clear that $\frac{d \mu}{d t}>0$ for all $t>0$, which is consistent with what was observed in Sec. III. For small $t$, we should then have

$$
\frac{d \mu}{d t} \approx \alpha \gamma k^{2} \theta(0)(\theta(0)-m)=\frac{\alpha \gamma k^{2}}{\sqrt{1+A^{2} k^{2}}}\left(\frac{1}{\sqrt{1+A^{2} k^{2}}}-\frac{U}{k \gamma}\right),
$$

while for large $t$ we should have

$$
\frac{d \mu}{d t} \approx \lim _{t \rightarrow \infty} \alpha \gamma k^{2} \theta(t)(\theta(t)-m)=\alpha \gamma k^{2}\left(1-\frac{U}{k \gamma}\right) .
$$

Both results are consistent with estimates obtained in Sec. III. Similar results can be obtained for $\beta(t)$ and $\omega(t)$ in terms of the function $\theta(t)$. Therefore, the dynamics of the system (21)-(23) can essentially be reduced to a study of the initial value problem (55) and (58).

With so much qualitative information gleaned from Eq. (55), it is natural to wonder whether an exact solution might be possible. Separating variables, one has

$$
\int \frac{d \theta}{F(\theta)}=\alpha \gamma k^{2} t+C_{0},
$$

where $C_{0}$ is an integration constant. Observe that

$$
\int \frac{d \theta}{F(\theta)}=\int \frac{d \theta}{\theta^{2}(\theta-m)\left(1-\theta^{2}\right)}=\frac{1}{m \theta}-\frac{\ln (\theta)}{m}-\frac{\ln (1+\theta)}{2(1+m)}-\frac{\ln (1-\theta)}{2(1-m)}+\frac{\ln (\theta-m)}{m^{2}\left(1-m^{2}\right)} .
$$

Therefore, to obtain a closed-form solution $\theta(t)$, we would need to solve the equation

$$
\frac{1}{m \theta}-\frac{\ln (\theta)}{m}-\frac{\ln (1+\theta)}{2(1+m)}-\frac{\ln (1-\theta)}{2(1-m)}+\frac{\ln (\theta-m)}{m^{2}\left(1-m^{2}\right)}=\alpha \gamma k^{2} t+C_{0}
$$

for $\theta$ as a function of $t$ (which we cannot do). Therefore, it is not possible to obtain an exact closed-form expression for $\theta(t)$ (and, therefore, for the solution of the system (21)-(23)). Hence, the qualitative and numerical results discussed here and in Sec. III are the best we can obtain when studying the temporal decay of Kelvin waves along a quantum vortex filament under the model of Schwarz.

\section{CONCLUSIONS}

For the eternal solutions (which do not decay in time), there is a requirement that the wave number is fixed. In other words, for fixed values of the parameters $A, \gamma, U$ there is a unique wave number $k=k^{*}$ given by (15) such that a constant-amplitude helical filament solution exists. This restriction in turn implies that the amplitude of such a helical filament must be bounded like $A<$ $\gamma /|U|$. This still allows the amplitude to be large enough for the helix to exhibit translational motion in addition to pure rotation, as is evident from the solution form presented in (18). The dispersion relation is close to that obtained in Ref. 15, though the dispersion relation $\omega$ obtained here is exact, while that of Ref. 15 was approximate (since an approximate potential form of the quantum LIA was assumed).

Note that the constant amplitude solution persists for all time, and that the mutual friction parameters $\alpha$ and $\alpha^{\prime}$ do not qualitatively influence this solution. This solution therefore connects the quantum LIA solutions back to the classical LIA helical solutions, since under the classical LIA there can be no decay or growth of the amplitude of (helical) solutions in time. In light of the DonnellyGlaberson instability, one can reason that solutions of this type for the classical LIA are a critical limit of those solutions to the quantum LIA. Yet, potential forms of the quantum LIA do not permit such time decay (at least in the helical filament case), so such solutions to approximating potential forms of the LIA are, in a sense, classical approximations for the quantum case, corresponding to solutions which maintain their form in time. 
For the solutions which are permitted to decay in time, some observations are in order. The rate of decay of the filament, $\mu(t) / t$, is not constant in time but rather varies according to (23). We see from the asymptotics of $\mu$ that the greatest rate of decay is for large time, so the helical perturbations along the filament decay slowly at first and more rapidly as time progresses. Since the translational effects are tied to the decay rate of the filament through a term of the form $\exp (-2 \mu(t))$, the greatest translation of the filament occurs for small time, while translation of the filament ceases for asymptotically large time. This makes sense: as the helical filament decays into a line filament, translational effects diminish since there is no translation of a line filament. This is demonstrated in Figure 4. Therefore, the Kelvin waves rotate and also move along the $x$-axis while decaying. The more they decay in amplitude, the slower the motion along the $x$-axis. Meanwhile, the rotational motion persists, even as the waves decay.

In the case of constant amplitude helical waves along a filament (i.e., the eternal solutions), the wave number is fixed (at a value corresponding to the Donnelly-Glaberson instability), while when the helical filament is allowed to decay to a line filament we have no such restriction on the wave number. However, we do still have a restriction on the possible value of $k$ which permit the filament to decay, $k / \sqrt{1+A^{2} k^{2}}>\frac{U}{\gamma}$. Rearranging this, we obtain a condition on $A: A<\sqrt{\frac{\gamma^{2}}{U^{2}}-\frac{1}{k^{2}}}<\frac{\gamma}{|U|}$. Therefore, the restriction on the amplitude is stronger when we permit the helical waves to decay. This is physically reasonable, as it is sensible to describe small-amplitude Kelvin waves along a vortex filament using LIA. For larger-amplitude perturbations, non-local effects will influence the solutions, so the full Biot-Savart law under the HVBK model would be more reasonable for describing the filament dynamics, as described in Sec. I. Even then, particularly large amplitude solutions are not particularly interesting, since by the Donnelly-Glaberson instability, such solutions should amplify (until they become structurally unstable and break up). Therefore, in the physically interesting case of small amplitude Kelvin waves, we have been able to obtain results demonstrating the manner of decay of Kelvin waves which are driven by the normal fluid flow directed along a quantum vortex filament.

If one is interested in multiple vortex filaments in close proximity (particularly in cases of quantum vortex filaments which may intersect) or strong turbulence, then the non-locality of the Biot-Savart formulation is needed, since the LIA is useful for individual isolated filaments (or for multiple filaments, in the case where each filament is sufficiently far removed from the others so that the self-induced motion of each has a negligible effect on the others). One possible area of future analytical work could be the dynamics of Kelvin waves along two quantum filaments which are permitted to intersect or to interact in close proximity. Since the LIA disregards the interaction of Kelvin waves propagating along the same vortex line, attempting to model this scenario analytically under growth/decay would also be interesting. Since the LIA best approximates the dynamics of Kelvin waves when the helix is not tightly coiled (i.e., when the wave number is not too large), it would be interesting to apply the non-local model (3) to the case where the wave number is large. This has been done for the classical vortex filament model (2) (where it was shown that in the $k \rightarrow \infty$ limit, the helix reduces to a cylindrical vortex sheet, see Ref. 23) but not for the quantum model (3). Again, the LIA is useful for cases where the filament is not tightly coiled. In a submitted work, we study the classical Biot-Savart helix, and obtain quantitative estimates in order to determine when the Biot-Savart integral and LIA solutions disagree. Similar results will hold for the quantum case, since both the classical and quantum formulations share the same non-local term.

\section{ACKNOWLEDGMENTS}

R.A.V.G supported in part by NSF Grant No. 1144246. The author wishes to thank the anonymous referees, whose comments have led to definite improvement in the presentation of the results.

\footnotetext{
${ }^{1}$ H. E. Hall and W. F. Vinen, "The rotation of liquid helium II. I. Experiments on the propagation of second sound in uniformly rotating helium II," Proc. R. Soc. London, Ser. A 238, 204 (1956a).

${ }^{2}$ H. E. Hall and W. F. Vinen, "The rotation of liquid helium II. II. The theory of mutual friction in uniformly rotating helium II,” Proc. R. Soc. London, Ser. A 238, 215 (1956b).

${ }^{3}$ I. L. Bekarevich and I. M. Khalatnikov, "Phenomenological derivation of the equations of vortex motion in He II," Sov. Phys. JETP 13, 643 (1961).
} 
${ }^{4}$ S. K. Nemirovskii, "Quantum turbulence: Theoretical and numerical problems," Phys. Rep. 524, 85 (2013).

${ }^{5}$ K. W. Schwarz, "Three-dimensional vortex dynamics in superfluid ${ }^{4} \mathrm{He}$ : Line-line and line-boundary interactions," Phys. Rev. B 31, 5782 (1985).

${ }^{6}$ L. D. Landau and E. M. Lifshitz, Fluid Mechanics (Addison and Wesley, 1959).

${ }^{7}$ G. Boffetta, A. Celani, D. Dezzani, J. Laurie, and S. Nazarenko, "Modeling Kelvin wave cascades in superfluid helium," J. Low Temp. Phys. 156, 193 (2009).

${ }^{8}$ C. F. Barenghi, M. Tsubota, A. Mitani, and T. Araki, "Transient growth of Kelvin waves on quantized vortices," J. Low Temp. Phys. 134, 489 (2004).

${ }^{9}$ D. K. Cheng, W. M. Cromar, and R. J. Donnelly, "Influence of an axial heat current on negative-ion trapping in rotating helium II," Phys. Rev. Lett. 31, 433 (1973).

${ }^{10}$ W. I. Glaberson, W. W. Johnson, and R. M. Ostermeier, "Instability of a vortex array in He II," Phys. Rev. Lett. 33, 1197 (1974).

${ }^{11}$ R. M. Ostermeier and W. I. Glaberson, "Instability of vortex lines in the presence of axial normal fluid flow," J. Low Temp. Phys. 21, 191 (1975).

${ }^{12}$ S. Kida, "A vortex filament moving without change of form," J. Fluid Mech. 112, 397 (1981)

${ }^{13}$ E. B. Sonin, "Dynamics of helical vortices and helical-vortex rings," EPL 97, 46002 (2012).

${ }^{14}$ R. A. van Gorder, "Fully nonlinear local induction equation describing the motion of a vortex filament in superfluid ${ }^{4} \mathrm{He}$," J. Fluid Mech. 707, 585 (2012).

${ }^{15}$ R. A. van Gorder, "Motion of a helical vortex filament in superfluid ${ }^{4} \mathrm{He}$ under the extrinsic form of the local induction approximation," Phys. Fluids 25, 085101 (2013).

${ }^{16}$ R. A. van Gorder, "Quantum vortex dynamics under the tangent representation of the local induction approximation," J. Fluid Mech. 740, 5 (2014).

${ }^{17}$ N. Hietala and R. Hänninen, "Comment on 'Motion of a helical vortex filament in superfluid $4 \mathrm{He}$ under the extrinsic form of the local induction approximation' [Phys. Fluids 25, 085101 (2013)],” Phys. Fluids 26, 019101 (2014).

${ }^{18}$ R. A. van Gorder, "Response to "Comment on 'Motion of a helical vortex filament in superfluid $4 \mathrm{He}$ under the extrinsic form of the local induction approximation"” [Phys. Fluids 26, 019101 (2014)]," Phys. Fluids 26, 019102 (2014).

${ }^{19}$ M. Tsubota, T. Araki, and C. F. Barenghi, "Rotating superfluid turbulence," Phys. Rev. Lett. 90, 205301 (2003).

${ }^{20}$ W. F. Vinen, "Decay of superfluid turbulence at a very low temperature: The radiation of sound from a Kelvin wave on a quantized vortex," Phys. Rev. B 64, 134520 (2001).

${ }^{21}$ T. Araki, M. Tsubota, and S. K. Nemirovskii, "Energy spectrum of superfluid turbulence with no normal-fluid component," Phys. Rev. Lett. 89, 145301 (2002).

${ }^{22}$ S. Z. Alamri, A. J. Youd, and C. F. Barenghi, "Reconnection of superfluid vortex bundles," Phys. Rev. Lett. 101, 215302 (2008).

${ }^{23}$ R. L. Ricca, “The effect of torsion on the motion of a helical vortex filament,” J. Fluid Mech. 273, 241 (1994). 